\title{
Robots as Embodied Beings - Interactionally Sensitive Body Movements in Interactions Among Autistic Children and a Robot
}

\author{
Ben Robins \\ Adaptive Systems Research Group \\ School of Computer Science \\ University of Hertfordshire \\ Hatfield, Herts AL10 9AB, U.K \\ b.robins@herts.ac.uk
}

\author{
Paul Dickerson \\ School of Human and Life Sciences \\ Roehampton University \\ London, SW15 5PU, U.K \\ p.dickerson@rus.roehampton.ac.uk
}

\author{
Kerstin Dautenhahn \\ Adaptive Systems Research Group \\ School of Computer Science \\ University of Hertfordshire \\ Hatfield, Herts AL10 9AB, U.K \\ k.dautenhahn@herts.ac.uk
}

\begin{abstract}
The general context of the work presented in this paper is assistive robotics with our long-term aim to support children with autism. This paper is part of an investigation into what ways and to what extent a robot can assume the role of a social mediator - encouraging autistic children to interact with the robot, with each other and with co-present adults. The article provides a case study evaluation of segments of trials where four children with autism interacted with a robot as well as with each other. It focuses primarily on the ways in which the autistic children were found to skilfully orientate and reorientate their bodies in a way that was sensitive to the activities of the adult (such as requests and adjustments to the robot), the robot (its position and movement) and another child. Results are presented using an analysis of interaction informed by conversation analytic principles. The analysis showed how the children exhibited interaction skills where the robot served as a salient object mediating joint attention with other children.
\end{abstract}

\section{INTRODUCTION}

In recent years, software and robotic based interactive learning environments have been studied increasingly in the therapy or education of people with autism [1-5]. The work presented in this paper is part of the Aurora project, rooted in assistive technology and robot-human interaction research [6]. This project investigates the potential use of robots as therapeutic or educational 'toys' specifically for use by children with autism. The research focuses on ways that robotic systems can engage autistic children in simple interactive activities, such as turn-taking or imitative interactions. The overall aim is to encourage basic communication and social interaction skills.

In line with many other research activities in assistive robotics our work is strongly guided by the needs and preferences of individual subjects. This often involves working with a small group subjects in order to explore and evaluate the potential of a particular assistive robot and to assist its development, c.f. [7]. Note, in assistive robotics the use of control groups is usually not relevant since robotic systems are being developed for the purpose of assistance, not as a tool in order to access how the specific target group differs from other subjects without the particular impairments of physical, mental or social functions concerned. We therefore specifically target children with autism as our user group, working on a long-term basis with a small group of children with autism ${ }^{*}$. Given the nature of autism (a spectrum disorder) which implies huge differences among the subjects, and the therapeutic/educational background, our work is guided by the individual needs and preferences of the children. Given this specific context, we conduct trials within a rather broad context (compared to studies in experimental HRI research), exploring the interaction space involving children with autism and a robot interacting in a familiar and relatively unconstrained environment. In our previous work, we used both quantitative [8-10] as well as qualitative evaluation techniques [11]. The former adapted methods of quantitative observational analysis commonly used in psychology and ethology. The latter included Conversation Analysis [11]. In this paper we present a case study evaluation. Results are presented using an analysis of interaction informed by conversation analytic principles [11]. Selected interaction sequences are presented in the form of video stills and detailed descriptions of the interactions in light of the role of the robot as a social mediator.

This paper continues our research into joint attention skills in triadic interactions involving a robot, a child, and a second person. We investigate the ways and the extent a robot, being an object of joint attention, can assume the role of a social mediator encouraging autistic children to interact with the robot, with each other and with co-present adults. Previously the second person involved was an adult (the experimenter) [11]. The current work includes a scenario where not only the adult experimenter, but also a second child with autism was present.

\section{A. Autism}

Autism is a lifelong developmental disability that affects the way a person communicates and relates to people around them. The main impairments that are characteristic of people with autism lie in the areas of social interaction, communication and imagination [12]. People with autism usually exhibit little reciprocal use of eye-contact and rarely get engaged in interactive games. They have difficulties in understanding gestures and facial expressions, difficulties with verbal and non verbal communication, and are usually

\footnotetext{
* This approach is different from large-scale experimental user studies with existing robotic platforms in Human-Robot Interaction research, aiming at statistically determining differences between experimental conditions, involving control groups.
} 
impaired in understanding others intentions, feelings and mental states.

The exact causes of autism are still unknown, and at present no cure exists. A variety of therapeutic and educational approaches are known. Any such therapeutic or educational contribution benefits some, but not all children with autism. Our approach to use robots is hoped in future to serve a complementary role: exploiting the fact that children with autism, like most children, show a great affinity towards robots, and using the robot as a useful and programmable toy [3].

\section{B. Joint Attention in Autism}

From infancy, children use non-verbal interactive resources such as eye-gaze and protodeclarative pointing to share their attention and interest in an object or a third person with others. These triadic referencing activities are referred to as joint attentional skills and play a crucial role in the development of autistic children. Impairment in these skills are among the earliest abnormalities noticed in autism [13-15].

Increasingly, researchers are using robotic systems to study the development of social skills in children with autism. Fasel et al. [16] used simulated and robotic systems to explore the development and dysfunction of shared (joint) attention in toddlers with and without autism. Kozima and Yano, working with a robot that can create and maintain basic joint attention with a human, proposed the development of games that autistic children could play and possibly learn social interaction skills [1].

\section{Current work}

Previous research in the Aurora project illustrated the ability of a mobile robot to provide a focus of attention, and shared attention, in trials with pairs of children with autism [17]. More recently, Robins et al. explored robot-mediated joint attention in children with autism using a small humanoid robotic doll [11]. Extending the findings from these previous investigations, this article provides a case study evaluation, of segments of trials where four children with autism interacted with a robot as well as with each other, using the robot as a mediator, an object of joint attention. We focus our analysis primarily on the ways in which the autistic children were found to skilfully orientate and re-orientate their bodies in a way that was sensitive to the activities of the adult (such as requests and adjustments to the robot), the robot (its position and movement) and the other child. Such issues of body kinesics on the role and timing of nonverbal behaviour, including body movements, in communicative and interactional dynamics, play a fundamental part in humanhuman interaction and have also been highlighted recently as a challenge in human-robot interaction [10].

The focus on a sequentially sensitive analysis of the autistic children's activities enables us to investigate the ways in which such re-positioning of their bodies, as opposed to being indifferent to the context, are remarkably finely tuned with the changing moment by moment features of their interactional context. The analysis showed how the children exhibited interaction skills where the robot served as a salient object mediating joint attention with other children.

\section{THE TRIALS}

The trials took place in a special school for children with moderate learning difficulties in Hertfordshire, UK, which also has a small base for children with autism ${ }^{\dagger}$.

As stated above, the aim of the current study was to investigate how the robot can mediate interaction amongst children with autism. The trials were designed to allow pairs of children to play with the robot at the same time, with the hope that the robot, being an object of shared attention, will mediate and encourage the children to interact with it and with each other. In order to minimize any possible anxiety that the children might experience, being in a novel situation with a new and unusual toy (the robot) and a new person (the investigator), each child participated in few preliminary trials with the robot, on their own, without a second child present. These pre-trials were designed to allow the children to get used to the presence of the investigator, get familiar with the robot and to have unconstrained interaction with the robot with a high degree of freedom. We wanted to provide a reassuring environment where the repetitive and predictable behaviour of the robot is a comforting factor. In the main trials of this study, we have continued with the same approach where the children continue to have opportunities for free and unconstrained interactions with the robot and with each other.

\section{A. The Robot}

The robot used in these trials is Robota- a humanoid robotic doll (see figure 1).

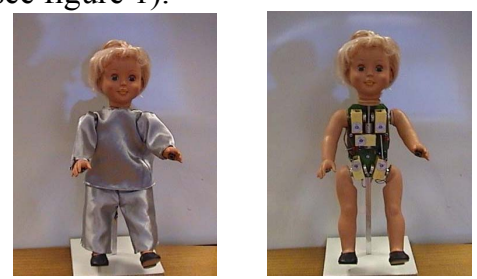

Figure 1 The robot Robota. The figure on the right shows the 'undressed' version revealing the robotic parts that control its movement

The robot's main body contains the electronic boards and the motors that drive the arms legs and head giving 1 DOF to each. The head and limbs are plastic components of a commercially available doll. Robota has the capability to connect to an array of various sensors, and to support a spectrum of multi modal interactions with children. For a complete description of Robota's hardware see [18].

The robot's features of speech processing, learning and motion tracking were not used in current trials and its operation and behaviour have been greatly simplified. This was necessary

\footnotetext{
We are grateful to the headteacher, teaching staff, parents and children at Middleton School. Special thanks go to the head of autism provision Mrs. Philp for her continued support. We would like to thank Aude Billard at EPFL for her supportive collaboration in the context of using the humanoid robot developed by her in our trials.
} 
due to the children's impairment (e.g. severely impaired speech, inability to be still and have long enough focus of attention, and maintaining gaze on another face etc.). In our trials, the robot has been programmed to operate as a puppet, whereby the investigator is the puppeteer and, unknown to the children, moves the robot's arms, legs and head by simple press of buttons on his laptop (Wizard of Oz approach), cf. [8].

Based on the results of our previous study into robot appearance suitable for children with autism, [8,9], the robot was dressed in a plain costume, and had simplified head features (i.e. short simple hair style, plain, lips and deemed colour eye-lashes).

\section{B. Trials set-up \& procedures}

The trials were conducted in a familiar room often used by the children for various activities. The room size was approximately $2.5 \mathrm{~m}$ with a carpeted floor. The room is an internal room and had one door and one window overlooking an open plan area with other class activities. The robot was positioned on a table and connected to a laptop. The investigator was sitting next to the table operating the laptop when necessary. Two stationary video cameras were used to record the trials. The children were brought into the room two at a time, by the investigator who collected them from their classroom. Each trial lasted as long as the children were comfortable with staying in the room. The trials stopped if the children indicated they wanted to leave the room or if they had stopped all interactions and got bored after spending at least 3 minutes already in the room. The average duration of each trial was approximately 5 minutes. The study expanded over several months and trials were designed to progressively move from very simple exposure to the robot to more complex opportunities for the children to get engaged in interactions with each other. During the later trials, the investigator verbally encouraged the children to show each other how they could interact with the robot. This was necessary in order to bootstrap the engagement of the children with the robot. In previous trials [10], this same effect was achieved without such explicit verbal instructions, but it required a longitudinal approach where the children could discover interactions with the robot in their own time. Since the current work was intended to focus on the robot's role of a mediator, we decided to use the explicit means of verbal encouragement which was applicable to the particular group of children we worked with.

\section{RoBOtS AS EMBODIED BEINGS - A CONTEXT FOR AUTISTIC CHILDREN TO DisPlay SOPHISTICATED EMBODIED ACTIONS}

In this analysis we studied ways in which the autistic children were found to skilfully orientate and re-orientate their bodies in such a way that was responsive to the activities of the adult (e.g. giving the children requests or instructions) and the robot (its position and movement). It involved the initiation of new actions as well as physical contact between the children, both of them were unexpected occurrences, given the children's skills exhibited in other contexts.
The robot demonstrated its role as a social mediator, an embodied being ${ }^{t}$ in the sense of providing an interactive context where social skills in children with autism were facilitated and encouraged. The concept of using a robot as a social mediator to facilitate interactions among people was initially proposed in [19], and first examples were documented in [17],[11]. This work is complementary to research into developing sociable robots that can ultimately possess sophisticated social skills $[20,21]$. The robot's behaviour was sensitive to even subtle changes in the children's behaviour, controlled via the experimenter. This sensitivity, that goes beyond current state of the art sensor processing abilities and control for robots, as well as its physical presence and interactivity afforded a social play context where the autistic children displayed sophisticated embodied actions and interactions.

\section{A. Responsiveness to adult requests}

The following example is taken from a trial where the investigator tried to encourage the children to play a game whereby the robot will not move unless the children will together show a movement similar to the robot's. Note, the typical interaction pattern with the robot usually involved lifting the arms or legs. Images 1-5 in figure 2 show the activities of the children during 4 seconds whilst nothing further was said by the investigator.

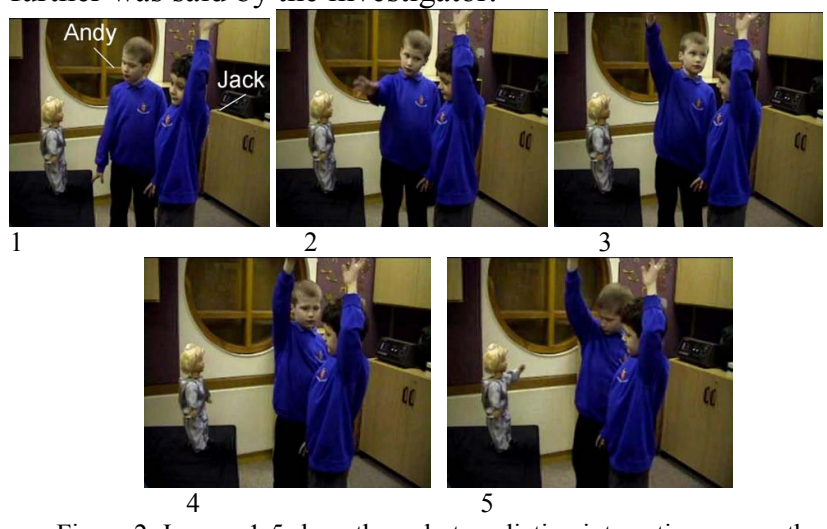

Figure 2: Images 1-5 show the robot mediating interaction among the children - example one.

We see how Jack ${ }^{\S}$ (on the right of image 1) made the first movement - whilst Andy first looked at the robot (1), then gazed at Jack (2) and started to imitate him, then he looked at the top of Jack's hand to ensure he is doing the same (3), then he gazed again on Jack's face (4) before looking if or how the robot responded (5) (and by then, the robot's arm was raised). This sequence shows the ways in which following an adult's request for the production of the same behaviour one child has co-joined the action of the other - with gaze playing a particularly important part in the synchronisation of their body movements.

\footnotetext{
"Note, we are using the term embodied 'being' for the robot referring to the situational, social context, we do not imply that the robot possesses sentience or any cognitive, emotional, or physiological properties characteristic of biological systems.

$\S$ Names of children used in this paper are synonyms.
} 


\section{B. Responsiveness to the robot}

(i) Responsiveness to the robot's position

In addition to moving appropriately in response to a request the children are also shown to position themselves appropriately even when not asked to do so by an adult. In figure 3 image 6, for example, Adam (right) positions himself such that he is aligned with Rob (left) facing the robot with his right side protruding - such that he is able to see the robot and raise his right arm without colliding with Rob.

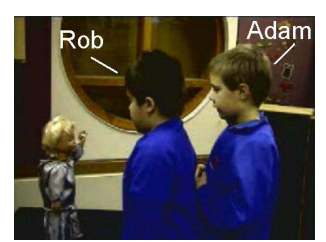

In this way both Adam and Rob are positioned such that they can monitor and interact with the robot without colliding with each other for the predicted range of activities which may follow.

6

Figure 3: Adam positions himself

\section{(ii) Responsiveness to the robot's movement}

As well as responding appropriately to requests and positioning their bodies appropriately vis a vis the robot and each other, the children were also found to respond to (or orientate to) the actions of co-present others (robot and child). Thus the movement of the robot - in particular the movement of its arm - is responded to by repositioning of body orientation and the enactment of gestures (figures 4 and 5 below).
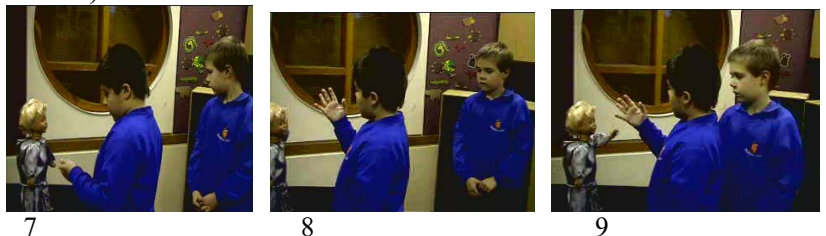

Figure 4: Adam attending to the actions of Rob and the robot

In image seven Adam is momentarily not attending to the robot, he is gazing to one side away from the direction of the robot. Rob is orientated in the general direction of the robot but is gazing specifically at his own hand. In image eight the robot has raised its hand and Rob has raised his hand. Adam now gazes at (or attends to) the area occupied by the robot and Rob. In image nine the robot and Rob are lowering their arms. Adam has moved closer to the robot.

In image ten the robot has started to raise its arm and Adam has swiftly straightened his arm. In image eleven the robot's arm is straightened and Adam and Rob both hold their 'mirrored' hands up.

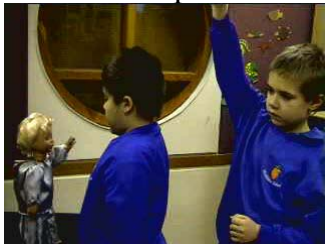

10

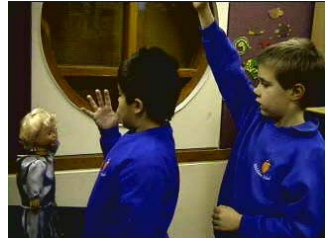

11
Figure 5: Adam and Rob 'mirroring' the robot

\section{Robot mediated initiated actions}

In this example the investigator tried to prompt both children to raise their hands at the same time. Andy pointed with his finger to his leg (12) wanting to include also the legs in the interaction game with the robot. Jack responded with a stretch of both, a leg and an arm, whilst Andy gazed at him (13). Andy then imitated Jack and looked at the robot (14), possibly to see if the robot respond in the same way?

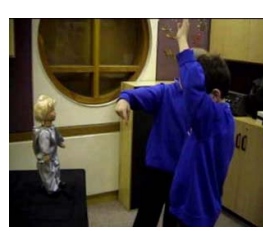

12

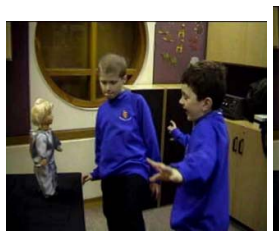

15

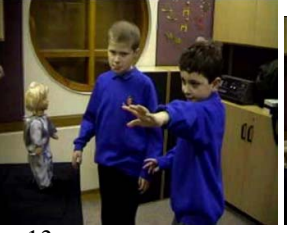

13

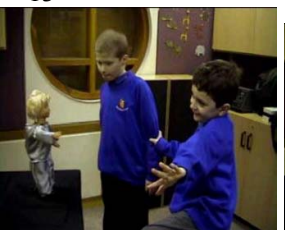

16

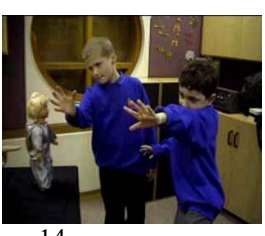

14

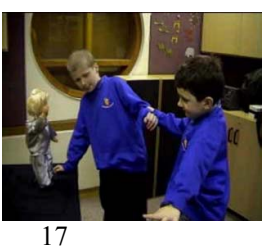

17
Figure 6: Stills 12-17 show a second example of the robot mediating interaction among the children

Andy and Jack then tried few stretches of hands and legs (e.g. image 15) and then they interacted with each other - using each other to balance themselves (images $16 \& 17$ ).

Stills 12-17 in figure 6 above show also how the embodied form of the robot differs, in the possibilities it provides, from a two dimensional representation (e.g. on a computer screen). In the example above the robot encouraged and provided an opportunity for a full body experience for the children, stretching themselves and exploring their own balances as well as experiencing each other in their interaction.

\section{Robot mediated physical contact between the children}

In the sections above we showed examples how the robot provided a context in which the autistic children displayed embodied sophistication in three separate aspects:

- How they orientated their body as a response to an adult's request (the investigator),

- how they positioned and repositioned their own body in relation to the static and moving robot, and

- how they initiated actions (such as leg movement) in the context of interacting with the robot and each other.

The following sections analyse how, when putting all these three aspects together, the embodied robot provides a context that might encourage one child to interact with another in a physical way (touch). This behaviour is very common amongst typically developing children - but is very unusual amongst children with autism, and even more so with the particular child concerned.

The focus in the following example is on Adam, who during previous trials showed a keen interest in the robot. It is 
also important to know that information gathered during special observation sessions (which has also been confirmed by his teachers) suggests that Adam doesn't show much interest in other children in his class, nor in their activities. During class activities Adam constantly tries to avoid the rest of the children - positioning himself, at any opportunity he can, in the corner of the room standing with his back to the rest of the children, or trying to escape from the room all together. This repeats itself in the playground during breaks, where Adam can always be found at the perimeter of the play area, most of the time standing, or pacing a few steps, but with his back to the rest of the children.

In contrast to what appears as lack of interest in his class mates, Adam showed a keen interest in the robot. In a previous trial where he was alone with the robot (only the experimenter present) he interacted with it for nearly the whole duration of the trial. In a different previous trial where he was with another child, he sat at the side: watching the robot and what was done to the robot when the other child interacted with it.

The following examples (figures 7 - 12) are different sections of one trial where Adam had been brought together with Rob to the room and both were encouraged to interact with the robot and with each other.

\section{(i) Example 1}

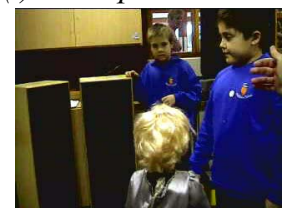

As Adam initially stayed at the back of the room, the investigator called him (image 18) saying "Adam come closer and look what Rob is doing".

18

Figure 7 -Adam stayed at the back

Adam responded to the request and came closer. Rob, at that time, was not actively engaged with the robot and the investigator prompted him to show Adam what the robot is capable of doing. Immediately following this request Adam held Rob's hand (image 19) - an action which at a bear minimum can be seen as in some way responsive to the proximity of Rob. Rob in turn responded by coming closer to the robot (image 20), and Adam followed him (image 21).

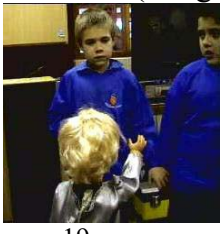

19

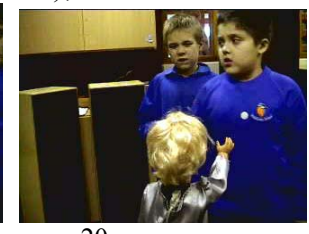

20

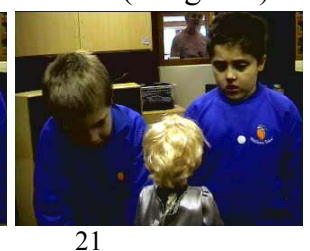

21
Figure 8: Adam held Rob's hand during the interactionjj

\section{(ii) Example 2}

After a while, when Rob still did not demonstrably engage with the robot, the investigator encouraged Adam to show Rob how to interact with the robot. In an action which appears responsive to this request Adam moved closer to Rob and put his arm on Rob's shoulder (image 22). Rob at this point started to imitate the robot's hand movement, and Adam, noticing this, turned towards the robot and touched the robot's hand (23).

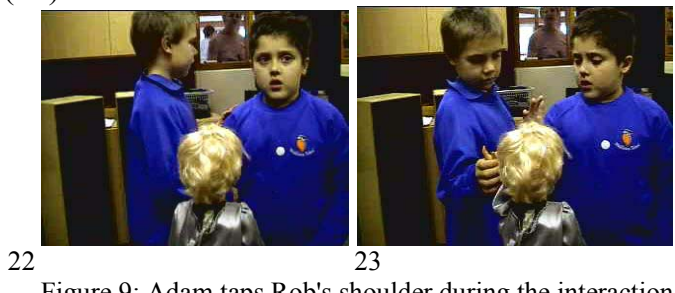

Figure 9: Adam taps Rob's shoulder during the interaction

\section{(iii) Example 3}

Adam and Rob continued to interact with the robot, following the investigator's prompts. A little bit later, after watching Rob's response, and when the investigator stopped prompting - Adam turned his back to the robot, (image 24) an action which if taken out of context might confirm notions regarding the asociability of autistic children in general and Adam in particular. However what is particularly striking is the way in which Adam's position still enabled him to monitor both Rob and the robot by turning his head - which he did at crucial moments such as when the robot's motor made a noise indicating movement (25). Such monitoring actions occurred at crucial moments in terms of the robot's activities (images $25 \& 27$ ) and occurred within the context of Adam's body being positioned such that it faced away from both Robot and Rob - yet sufficiently close to both to hear the robot and (as is shown in image 28) to touch Rob.
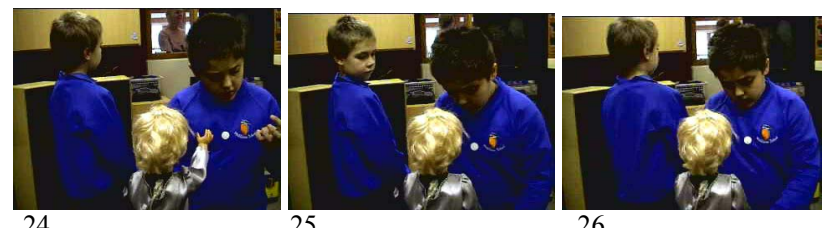

Figure 10: Adam is able to monitor Rob and the robot

In image 28 Adam engages in an action that would not be possible had his body been positioned further away - that is, with his back to the robot, Adam put his hand on Rob's shoulder, paused at this position for about 4 seconds and moved away (29).

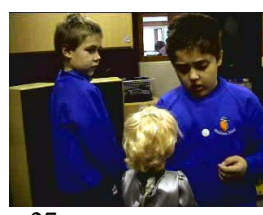

27

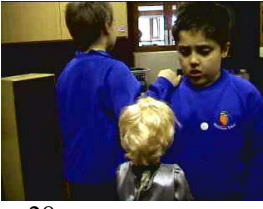

28

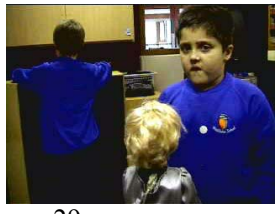

29
Figure 11: Adam put his hand on Rob's shoulder

(iv) Example 4

Immediately after the above, the investigator called Adam to return. Adam oriented himself towards the space behind Rob (image 30) and then positioned himself directly behind Rob and paused there for several seconds to watch Rob interacting with the Robot (image 31). 
Adam then touched Rob on both shoulders (image 32) in a way which could be seen as responsive to the alignment of Rob and the robot that he was facing. Next, Adam stepped to one side such that both children were now facing the robot and could monitor and respond to its movements and Adam could (as he subsequently did) monitor Rob interacting with the robot using simple imitative movements (image 33). Adam stayed in this position for a while before moving away.

It is important to point out here, that according to the teachers, the behaviour of Adam displayed in these examples - e.g. not only being so physically close to another child, but touching, and leaning against another child, was very unusual, and they could not recall any prior occasions where he had behaved in this way towards any other child.

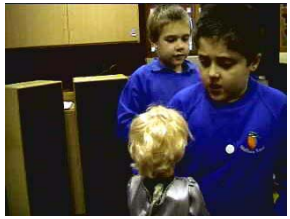

30

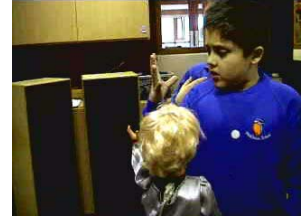

31

Figure 12 Adam watching Rob's interaction with the robot
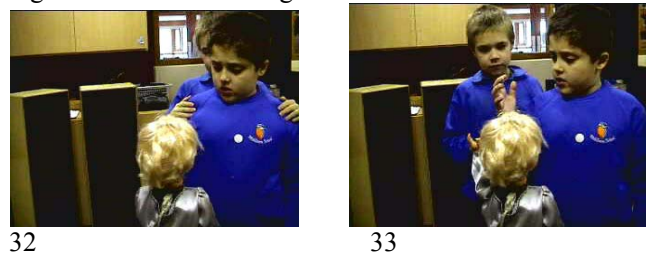

Figure 13: Adam touching Rob on both shoulders

\section{CONCLUSION}

In this paper we presented a case study evaluation using an analysis of interaction informed by conversation analytic principles [11]. Results highlighted different ways where the robot provided a context in which the autistic children displayed an embodied sophistication - they orientated their bodies a) in response to a request from the investigator, $b$ ) to the robot with regards to its position and its movement $\mathrm{c}$ ) to initiate new body movements and d) to each other's bodies using touch. These findings highlight the advantage of using an embodied robot rather than a computer simulation - the embodied nature of the robot allowed for the displays of such body orientation and full body experience in ways that a twodimensional display on a computer screen is unlikely to evoke. In addition, the robot's role as an object of shared focus of attention was displayed throughout the sophisticated embodied actions of the children. The robot became an embodied entity which allows for an exploration of how children position themselves with regards to it and each other - and as such an excellent tool for exploring how they might interact with other embodied entities such as humans e.g. other children.

\section{REFERENCES}

[1] H. Kozima and H. Yano, "Designing a robot for contingency-detection game," Proc. Workshop on Robotic and Virtual Interactive Systems in Autism Therapy, 2001.

[2] K. Dautenhahn and I. Werry, " Issues of robot-human interaction dynamics in the rehabilitation of children with autism," Proc. The Sixth
International Conference on the Simulation of Adaptive Behavior (SAB2000). 11 - 15 September 2000., Paris, France, 2000.

[3] K. Dautenhahn and I. Werry, "Towards interactive robots in autism therapy: Background, motivation and challenges," Pragmatic and Cognition, vol. 12, pp. 1-35, 2004.

[4] F. Michaud, A. Duquette, and I. Nadeau, "Characteristics of mobile robotics toys for children with pervasive developmental disorders.," Proc. IEEE international conference on Systems, Man and Cybernetics, 2003.

[5] D. Wada, T. Shibata, T. Saito, and K. Tanie, "Analysis of factors that bring mental effects to elderly people in robot assisted activity," Proc. Int Conference on Intelligent Robots and Systems, IROS 2002, Lausanne, Switzerland, 2002.

[6] AURORA, URL: http://www.aurora-project.com/last accessed 03/30/05, 2005.

[7] M. Hillman, "Rehabilitation robotics from past to present - a historical perspective," Proc. Eighth International Conference on Rehabilitation Robotics, ICORR, 2003, 2003.

[8] B. Robins, K. Dautenhahn, and J. Dubowski, "Investigating Autistic Children's Attitudes Towards Strangers with the Theatrical Robot-A New Experimental Paradigm in Human-Robot Interaction Studies?," Proc. 13th IEEE International Workshop on Robot and Human Interactive Communication - RO-MAN,Kurashiki, Japan, 20-22 September, 2004.

[9] B. Robins, K. Dautenhahn, R. t. Boekhorst, and A. Billard, "Robots as Assistive Technology - Does Appearance Matter?," Proc. 13th IEEE International Workshop on Robot and Human Interactive Communication - RO-MAN, Kurashiki, Japan, 20-22 September, 2004.

[10]B. Robins, K. Dautenhahn, R. te-Boekhorst, and A. Billard, "Robotic assistants in therapy and education of children with autism: can a small humanoid robot help encourage social interaction skills?," Universal Access in the Information Society, vol. 4:2 (in press), 2005.

[11]B. Robins, P. Dickerson, P. Stribling, and K. Dautenhahn, "Robotmediated joint attention in children with autism: A case study in a robothuman interaction," Interaction studies: Social Behaviour and Communication in Biological and Artificial Systems, vol. 5:2 John Benjamins Publishing Company, Amsterdam,pp. 161-198, 2004.

[12]NAS, " National Autistic Society UK, url: http://www.nas.org.uk, last accessed 03/30/05," 2005.

[13]T. Charman, "Why is joint attention a pivotal skill in autism?," Philosophical Transaction: Biological Science, vol. 358 The Royal Society, London, pp. 315-324, 2003.

[14]M. Siller and M. Sigman, "The behaviours of parents of children with autism predict the subsequent development of their children's," Communication Journal of Autism \& Developmental Disorders, vol. 32(2), 77-89, 2002.

[15]R. Leekam, "Pointing and showing problems for autistic children," in Press release on an ESRC funded research report.'Dyadic Orienting and Joint Attention in Children with Autism' http://www.eurekalert.org/pub_releases/2003-07/esr-as070303.php (retrieved December 13th 2003), 2003.

[16]I. Fasel, D. Gedeon, J. Triesch, and J. Movellan, "Combining Embodied Models and Empirical research for Understanding the Development of Shared Attention," Proc. The 2nd International Conference on Development and Learning (ICDL'02), 2002.

[17]I. Werry, K. Dautenhahn, B. Ogden, and W. Harwin, "Can Social Interaction Skills Be Taught by a Social Agent? The Role of a Robotic Mediator in Autism Therapy," in Proc. CT2001, The Fourth International Conference on Cognitive Technology: Instruments of Mind, LNAI 2117, M. Beynon, C. L. Nehaniv, and K. Dautenhahn, Eds. Berlin Heidelberg: Springer-Verlag, 2001, pp. 57-74.

[18]A. Billard, "Robota: Clever Toy and Educational Tool," Robotics and Autonomous Systems,pp. (42):259-269, 2003.

[19]K. Dautenhahn, "Robots as social actors: Aurora and the case of autism," Proc. The Third International Cognitive Technology Conference CT99, August, San-Francisco, 1999.

[20]T. Fong, I. Nourbakhsh, and K. Dautenhahn, "A Survey of Socially Interactive Robots," Robotics and Autonomous Systems, vol. 42,pp. 143166, 2003.

[21]C. Breazeal, Designing sociable robots: MIT press, 2002. 